\title{
Association of Preoperative Risk Factors and Mortality in Older Patients following Emergency Abdominal Surgery: A Retrospective Cohort Study
}

\author{
Nadir Adnan Hacım ${ }^{1}$, Ahmet Akbaş ${ }^{1}$, Yigit Ulgen ${ }^{2}$, Talar Vartanoglu Aktokmakyan ${ }^{1}$, Serhat Meric ${ }^{1}$, Merve Tokocin $^{1}$, \\ Onder Karabay ${ }^{3}$, Gulcin Ercan ${ }^{1}$, Yuksel Altinel ${ }^{1}$ \\ ${ }^{1}$ Department of General Surgery, Bagcilar Training and Research Hospital, Istanbul, Turkey \\ ${ }^{2}$ Department of Pathology, Bagcilar Training and Research Hospital, Istanbul, Turkey \\ ${ }^{3}$ Department of General Surgery, Beykent University, Istanbul, Turkey
}

Corresponding Author:

Nadir Adnan Hacim, MD

Department of General Surgery,

Bagcilar Training and Research

Hospital, Merkez Mah. Dr. Sadık

Ahmet Cd. No:5, 34200 Istanbul,

Turkey

E-mail: adnanhcm@hotmail.com

ORCID:

https://orcid.org/0000-0002-3906-2538

Received: September 8, 2021

Revised: November 8, 2021

Accepted: November 30, 2021

\begin{abstract}
Background: Older patients undergoing emergency laparotomy have high morbidity and mortality rates. Preoperative risk assessment with good predictors is an appropriate measure in this population. Frailty status is significantly associated with postoperative outcomes in older adults. This study aimed to investigate the effect of preoperative risk factors and frailty on short-term outcomes following emergency surgery for acute abdomen in older patients. Methods: This study included older patients ( $\geq 65$ years of age) who underwent emergency abdominal surgery. We retrospectively analyzed their demographic and clinical variables and used the modified Frailty Index-11 to evaluate their frailty status. The primary outcome was the 30 -day mortality rate. We also analyzed risk factors of mortality in these patients. Results: The study included 150 patients with a median age of 74 years. The mortality rate was $17.3 \%(n=26)$. We observed significantly higher mortality rates in patients who were obese and who had higher American Society of Anesthesiology (ASA grades) $(p<0.05)$. Frailty status was worse in deceased group $(p<0.001)$, when compared to individuals who survived. Septic shock was associated with the development of mortality $(p<0.001)$. Multivariate regression analysis revealed that ASA grade was the only independent risk factor for mortality (odds ratio $=19.642 ; 95 \%$ confidence interval, 3.886-99.274; $\mathrm{p}<0.001)$. Conclusion: Older patients with obesity and frailty presenting with higher ASA grades and septic shock had the worst survival following emergency abdominal surgery. The ASA grade was an independent risk factor for mortality.
\end{abstract}

Key Words: Acute abdomen, Aged, Frailty, Mortality rate

\section{INTRODUCTION}

The life expectancy of the older adult population is increasing globally. ${ }^{1)}$ The number of emergency laparotomies performed for geriatric patients increases similar to other surgical procedures in an aging population. ${ }^{2)}$ Although the definition of older adult is controversial, many authors agree on an age over 65 years. ${ }^{3)} \mathrm{Pa}$ tients who undergo emergency laparotomy have high morbidity and mortality rates with additional high risks. ${ }^{4,5)}$ In addition, older patients undergoing abdominal surgery in emergency settings usu- ally require intensive care and longer hospital stays. Therefore, compared to younger patients, it is essential to provide a safe, beneficial, and effective surgical assessment system to evaluate medical risks of consequent illness and clinical competence in older patients.

Chronological age alone may not be a good predictor for poor outcomes in emergency operations of older adults. ${ }^{6,7)}$ Preoperative risk assessment is an appropriate measure for decision-making and preemptive resource allocation. ${ }^{4)}$ Previous studies assessed in detail morbidity and mortality rates in older patients following emer- 
gency laparotomy. ${ }^{8-10)}$ Several perioperative risk scoring systems have been developed to assess the outcomes in surgical patients in elective or emergent situations. ${ }^{5)}$ These systems of identifying high-risk patients and providing them with the appropriate level of care include the American Society of Anesthesiology (ASA) grades, Portsmouth Physiological and Operative Severity Score (P-POSSUM) for the enumeration of mortality and morbidity, American College of Surgeons National Surgical Quality Improvement Program (ACS-NSQIP) surgical risk calculator, and National Emergency Laparotomy Audit (NELA) risk prediction calculator. $^{4-6,11-14)}$

The strengths and weaknesses of these tools have been reported. ${ }^{15)}$ The major criticisms include various parameters that may not be routinely performed and the overestimation of mortality and morbidity. ${ }^{4,6)}$ Moreover, there remains no consensus regarding the reliability of these tools in geriatric populations subjected to emergency laparotomy. ${ }^{16)}$ Because of differences among institutions in their risk prediction of mortality, detailed analyses are needed to determine the reproducibility of the variables used for these tools. Thus, identifying more accurate predictors of mortality in older patients could help identify a surgical assessment tool. $^{11,16)}$

There is a lack of consensus on the definition of frailty in the literature, and this term has been used subjectively., ${ }^{4,12,17)}$ It often denotes unintentional weight loss, exhaustion, weakness, slow walking, low physical activity, and lack of phenotype. ${ }^{4,18)}$ The Clinical Frailty Scale has been used to apply a more consistent definition. ${ }^{6,17)}$ Because of the lack of a uniform definition and a tool to identify frailty in older patients, several tools have been proposed in different patient groups. Among these, the Clinical Frailty Scale has the advantage of being fast and straightforward to perform. ${ }^{17,18)}$ However, a prospective clinical observation is needed, which includes comorbidities, ability to perform specific activities of daily living, oxygen supplementation, and presence of a terminal illness. The modified Frailty Index-11 (mFI-11) comprises 11 possible comorbidities/deficits that can be recorded retrospectively. ${ }^{6)}$

As frailty is significantly reportedly associated with 90-day mortality and postoperative morbidity in patients undergoing emergency laparotomy, ${ }^{19)}$ we examined the effect of preoperative frailty on the loss of functional independence following emergency abdominal surgery in older adults.

The present retrospective cohort study aimed to investigate the associations of some preoperative predictive factors, including frailty, with morbidity, mortality, management strategies, and short-term outcomes of emergency surgery for acute abdomen in patients aged 65 years and older.

\section{MATERIAL AND METHODS}

\section{Study}

This retrospective cohort study included all consecutive older patients who underwent emergency abdominal surgery at Bagcilar Training and Research Hospital in Istanbul, Turkey, between February 2016 and January 2020. The Bagcilar Training and Research Hospital Local Ethics Committee approved the study (No. 2020.10.2.01.162). We conducted this study in accordance with the principles of the Declaration of Helsinki. Written consent could not be obtained due to the retrospective design of the study and the anonymity of the data.

\section{Patients}

We retrospectively searched all emergency general surgery admissions using the hospital information system and medical records to include patients aged $\geq 65$ years. We identified a total of 831 patients. The exclusion criteria were age $<65$ years on the day of surgery $(n=647)$ and incomplete data $(n=11)$. All types of gynecological and urological procedures in non-trauma or trauma surgery settings $(n=23)$ were also excluded. Thus, we evaluated data from 150 admissions.

We collected data on patient characteristics, including demographic data, body mass index (BMI), comorbidities, ASA score, perioperative features, and outcomes. The patients were grouped based on their age (65-74 or $\geq 75$ years). Clinical data, including evidence of sepsis and systemic shock, systolic blood pressure $(\mathrm{mmHg})$, and the lowest or highest values of the laboratory parameters in the initial 24-hour period, were obtained from emergency department records. Comorbidities included diabetes mellitus (DM), hypertension, coronary artery disease (CAD), chronic obstructive pulmonary disease (COPD), and chronic renal failure (CRF). We also recorded perioperative characteristics, diseases requiring surgical treatment, surgical approach, type of surgery, duration, length of intensive care unit (ICU) and hospital stays, postoperative complications, and readmission data.

Frailty status was evaluated using mFI-11. This 11-point scoring system includes 11 possible comorbidities and deficits (Table 1). As a comorbidity-dominant scoring system, it requires binary non-subjective responses. The score can also be calculated without phenotypic measures of the patients, such as exhaustion, slowness, and reduced physical activity. ${ }^{6}$ Each component is assigned one point with a total point range of $0-11$. Frailty status is categorized as frail (score $>3$ points), pre-frail (1-2 points), and absence of frailty ( 0 points). For analysis, we grouped the patients as frail (frail and pre-frail) and non-frail.

Clinical decisions, including the decision to undergo surgery 
Table 1. Modified Frailty Index-11 (mFI-11)

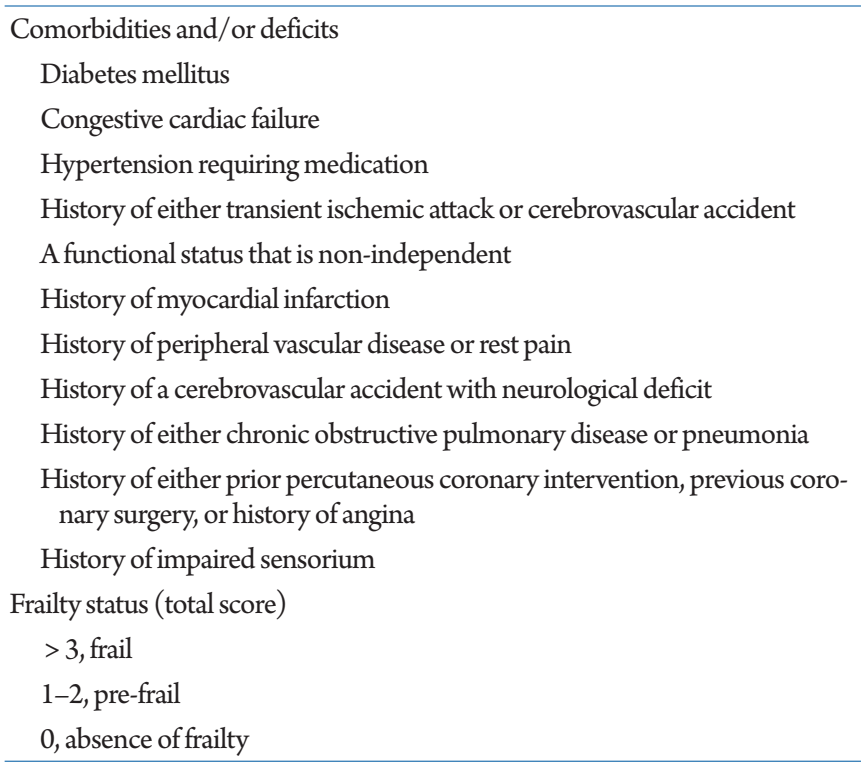

and choice of operative technique (open or laparoscopic), were based on the discretion of the attending consultant surgeons. The surgical procedures were performed by the consultant surgeons or under their supervision.

All patients were followed up to 30 days postoperatively or to death.

\section{Statistical Analyses}

The primary outcome was 30-day mortality. We grouped the patients based on the development of mortality (non-survivors and survivors).

We performed statistical analyses using SPSS version 15.0 (SPSS Inc., Chicago, IL, USA). Continuous variables with or without normal distribution are presented as mean \pm standard deviation and median with interquartile range (IQR, 25\% to $75 \%$ ). Categorical variables are presented as frequencies with percentages. We applied the Kolmogorov-Smirnov test to check the normality of the numerical variables. We used the independent samples t-test to compare two independent groups with normally distributed variables and the Mann-Whitney U test for non-normally distributed variables. We applied Pearson chi-square and Fisher exact tests in $2 \times 2$ tables to compare the differences in categorical variables.

Multivariate logistic regression analyses were performed to analyze the factors affecting the development of mortality. Statistically significant factors in the univariate analyses were included in the multivariate analysis. Statistical significance was set at $\mathrm{p}<0.05$.

\section{RESULTS}

Table 2 presents the details of the patients' demographic and clinical characteristics. The median age of the 150 patients was 74 years. Of these, 124 patients (82.7\%) survived to discharge (survivors), while 26 patients (17.3\%) died (non-survivors) within a median length of 3 days (IQR, 2-6).

The numbers of female and male patients were similar (71 vs. 79 ). The mean BMI of the study group was $26.7 \pm 4.2 \mathrm{~kg} / \mathrm{m}^{2}$. Most of the patients were classified as ASA III ( $\mathrm{n}=62 ; 41.3 \%)$, followed by ASA II ( $\mathrm{n}=46 ; 30.7 \%)$. Most patients $(90 \%)$ had at least one type of comorbid disease. Hypertension and DM were the two most common coexisting diseases in the study group. Frailty was not observed in 126 patients (84.0\%), 19 patients (12.7\%) were pre-frail, and five $(3.3 \%)$ were frail. Seventeen patients (11.3\%) experienced septic shock. Table 3 summarizes the laboratory findings of the study groups.

Gastrointestinal perforation was the most common diagnosis in the study group. Forty-four patients $(29.3 \%)$ had perforation, followed by intestinal obstruction in 30 patients $(20.0 \%)$. The other diagnoses are listed in Table 4. Intestinal resection with ostomy formation and anastomosis was the most frequently performed surgical procedure in 31 (20.7\%) and $22(14.7 \%)$ patients, respectively. The distribution of operations is presented in Table 5.

A total of 26 deaths occurred within postoperative 30 days (non-survivors), corresponding to a mortality rate of $17.3 \%$. The median age of the non-survivors was significantly higher than that of the survivors $(p<0.001)$. The rate of patients aged 75 years or more was significantly higher among the non-survivors than in the survivors $(80.8 \%$ vs. $41.9 \%)(\mathrm{p}<0.001)$. We observed significantly higher mortality rates in obese older patients with higher ASA grades (Table 3). Hypertension and CAD were more common in non-surviving patients $(\mathrm{p}=0.040$ and $\mathrm{p}=0.003$, respectively). The absence of frailty was more frequently observed in the surviving patients than in non-surviving patients $(91.1 \%$ vs. $50 \%$; $\mathrm{p}<0.001)$. All patients with frailty $(n=5)$ were non-survivors, whereas no patient in the survivor group was categorized as frail. Grouping based on frailty (frail and pre-frail) and the abscence of frailty revealed a significant difference in mortality rates $(54.2 \%$ vs. $10.3 \%$; $p<0.001)$. In non-survivors, the incidence of septic shock was higher than that in survivors $(\mathrm{p}<0.001)$.

Patients with frailty (frail and pre-frail) were likely to be older $(\mathrm{p}<0.001)$; have higher ASA grades $(\mathrm{p}<0.001)$; and have higher incidences of hypertension ( $\mathrm{p}=0.001), \mathrm{DM}(\mathrm{p}=0.046)$, and septic shock $(p=0.001)$. Other demographic and clinical variables were not significantly associated with frailty $(p>0.05)$.

We observed significant differences in laboratory parameters be- 
Table 2. Demographic and clinical characteristics of the study groups $(\mathrm{n}=150)$

\begin{tabular}{|c|c|c|c|c|}
\hline Variable & Overall $(n=150)$ & Non-survivors $(n=26)$ & Survivors $(\mathrm{n}=124)$ & $\mathrm{p}$-value \\
\hline Age (y) & $74(69-79)$ & $81(76-85)$ & $73(69-77)$ & $<0.001$ \\
\hline $65-74$ & $77(51.3)$ & $5(19.2)$ & $72(58.1)$ & $<0.001$ \\
\hline$\geq 75$ & $73(48.7)$ & $21(80.8)$ & $52(41.9)$ & \\
\hline Sex & & & & 1.0 \\
\hline Female & $71(47.3)$ & $12(46.2)$ & $59(47.6)$ & \\
\hline Male & $79(52.7)$ & $14(53.8)$ & $65(52.4)$ & \\
\hline $\operatorname{BMI}\left(\mathrm{kg} / \mathrm{m}^{2}\right)$ & $26.1(23.8-29)$ & $27.5(25.7-31.6)$ & $25.9(23.7-28.6)$ & 0.025 \\
\hline ASA grade & & & & $<0.001$ \\
\hline 1 & $10(6.7)$ & $0(0)$ & $10(8.1)$ & \\
\hline 2 & $46(30.7)$ & $1(3.8)$ & $45(36.3)$ & \\
\hline 3 & $62(41.3)$ & $2(7.7)$ & $60(48.4)$ & \\
\hline 4 & $26(17.3)$ & $17(65.4)$ & $9(7.3)$ & \\
\hline 5 & $6(4.0)$ & $6(23.1)$ & $0(0)$ & \\
\hline Comorbidity & & & & 0.470 \\
\hline Present & $135(90)$ & $25(96.2)$ & $110(88.7)$ & \\
\hline Absent & $15(10)$ & $1(3.8)$ & $14(11.3)$ & \\
\hline \multicolumn{5}{|l|}{ Types of comorbidity } \\
\hline Hypertension & $103(68.7)$ & $22(84.6)$ & $81(65.3)$ & 0.040 \\
\hline $\mathrm{DM}$ & $61(40.7)$ & $13(50)$ & $48(38.7)$ & 0.198 \\
\hline $\mathrm{CAD}$ & $25(16.7)$ & $10(38.5)$ & $15(12.1)$ & 0.003 \\
\hline COPD & $16(10.7)$ & $1(3.8)$ & $15(12.1)$ & 0.191 \\
\hline CRF & $14(9.3)$ & $4(15.4)$ & $10(8.1)$ & 0.206 \\
\hline Frailty & & & & $<0.001$ \\
\hline Absence of frailty & $126(84.0)$ & $13(50)$ & $113(91.1)$ & \\
\hline Pre-frail & $19(12.7)$ & $8(30.8)$ & $11(8.9)$ & \\
\hline Frail & $5(3.3)$ & $5(19.2)$ & $0(0)$ & \\
\hline Septic shock & & & & $<0.001$ \\
\hline Present & $17(11.3)$ & $12(46.2)$ & $5(4.0)$ & \\
\hline Absent & $133(88.7)$ & $14(53.8)$ & $119(96.0)$ & \\
\hline $\mathrm{SBP}(\mathrm{mmHg})$ & $140(120-160)$ & $135(90-150)$ & $145(120-160)$ & 0.075 \\
\hline
\end{tabular}

Values are presented as median (interquartile range) or number (\%).

BMI, body mass index; ASA, American Society of Anesthesiologists; DM, diabetes mellitus; CAD, coronary artery disease; COPD, chronic obstructive pulmonary disease; CRF, chronic renal failure; SBP; systolic blood pressure.

Table 3. Laboratory features of the study groups $(n=150)$

\begin{tabular}{lcccr}
\hline Variable & Overall $(\mathrm{n}=150)$ & Non-survivors $(\mathrm{n}=26)$ & Survivors $(\mathrm{n}=124)$ & $\mathrm{p}$-value \\
\hline Hemoglobin $(\mathrm{g} / \mathrm{dL})$ & $12.4(10.5-13.5)$ & $12.1(8.4-13.2)$ & $12.4(11.1-13.5)$ & 0.075 \\
WBC $\left(\times 10^{9} / \mathrm{L}\right)$ & $11.1(7.3-14.5)$ & $16.0(10.5-18.7)$ & $10.4(7.2-13.5)$ & 0.003 \\
Glucose $(\mathrm{mg} / \mathrm{dL})$ & $109(89-139.3)$ & $120(100-190)$ & $101(88-134)$ & 0.013 \\
Creatinine $(\mathrm{mg} / \mathrm{dL})$ & $1.4(1-3.1)$ & $3.6(1.9-4.4)$ & $1.3(1.0-1.75)$ & $<0.001$ \\
Sodium $(\mathrm{mEq} / \mathrm{L})$ & $138.8 \pm 4.6$ & $138.0 \pm 5.3$ & $139.0 \pm 4.5$ & 0.444 \\
Albumin $(\mathrm{g} / \mathrm{dL})$ & $3.9(2.9-4.9)$ & $2.35(2-3.4)$ & $4.15(3.3-4.95)$ & $<0.001$ \\
CRP $(\mathrm{mg} / \mathrm{dL})$ & $52.0(18.8-123.6)$ & $183.5(114-245)$ & $39(13.8-80.5)$ & $<0.001$ \\
NLR & $4.5(3.0-7.7)$ & $9.65(6.5-11.9)$ & $3.8(2.9-5.9)$ & $<0.001$
\end{tabular}

Values are presented as mean \pm standard deviation or median (interquartile range).

WBC, white blood cell; CRP, C-reactive protein; NLR, neutrophil-to-lymphocyte ratio. 
Table 4. Distribution of diagnoses in the study groups

\begin{tabular}{lccc}
\hline Diagnoses & Overall $(\mathrm{n}=150)$ & Non-survivors $(\mathrm{n}=26)$ & Survivors $(\mathrm{n}=124)$ \\
\hline Perforation & $44(29.3)$ & $16(61.6)$ & $28(22.6)$ \\
Intestinal obstruction & $30(20.0)$ & $2(7.7)$ & $28(22.6)$ \\
Mesenteric ischemia & $18(12.0)$ & $4(15.4)$ & $14(11.3)$ \\
Acute cholecystitis & $17(11.3)$ & $0(0)$ & $17(13.7)$ \\
Anastomotic problems & $12(8.0)$ & $1(3.8)$ & $8(8.9)$ \\
Acute appendicitis & $8(5.3)$ & $0(0)$ & $5(4.0)$ \\
Bleeding $^{\mathrm{a})}$ & $7(4.7)$ & $2(7.7)$ & $3(2.4)$ \\
Hernia $_{\text {Others }}^{\mathrm{b})}$ & $3(2.0)$ & $0(0)$ & $10(8.1)$ \\
\hline
\end{tabular}

Values are presented as number (\%).

${ }^{\text {a) }}$ Upper gastrointestinal $(\mathrm{n}=4)$, intra-abdominal $(\mathrm{n}=3) ;{ }^{\text {b) }}$ diagnostic laparotomy/laparoscopy $(\mathrm{n}=8)$, acute pancreatitis/gastrointestinal foreign body/invagination in each.

Table 5. Distribution of surgical procedures in the study group

\begin{tabular}{lc}
\hline Type of operation & $\mathrm{n}(\%)$ \\
\hline Intestinal resection with ostomy formation & $31(20.7)$ \\
Intestinal resection with anastomosis & $22(14.7)$ \\
Cholecystectomy & $17(11.3)$ \\
Diagnostic laparoscopy/laparotomy & $16(10.7)$ \\
Primary suturing & $16(10.7)$ \\
Ostomy formation & $15(10.0)$ \\
Adhesiolysis & $10(6.7)$ \\
Appendectomy & $8(5.3)$ \\
Others & $15(10.0)$ \\
\hline
\end{tabular}

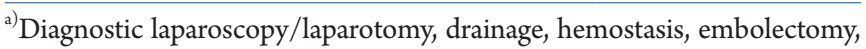
splenectomy, necrosectomy, hernia repair, and antrectomy.

tween the two groups (Table 3). Lower albumin values were significantly associated with the development of mortality $(\mathrm{p}<0.001)$. Non-survivors had significantly higher white blood cell (WBC) counts and glucose, creatinine, and C-reactive protein (CRP) values $(\mathrm{p}=0.003, \mathrm{p}=0.013, \mathrm{p}<0.001$, and $\mathrm{p}<0.001$, respectively). The median neutrophil-to-lymphocyte ratio (NLR) values differed significantly between the non-survivor and survivor groups ( 9.65 vs. $4.8 ; \mathrm{p}<0.001)$.

We observed significant associations between the diseases requiring surgical treatment and the development of mortality $(\mathrm{p}=0.004)$ (Table 4). Perforation was detected in $61.6 \%$ of the non-survivors compared to $22.6 \%$ of the survivors. No patients with acute cholecystitis or appendicitis died. Intestinal obstruction was more frequently observed in survivors ( $22.6 \%$ vs. $7.7 \%$, respectively). The disease-related mortality rates for perforation, bleeding (intra-abdominal or gastrointestinal), and mesenteric ischemia were $36.4 \%$, $28.6 \%$, and $22.2 \%$, respectively. The distribution of the most frequently performed surgical procedures is presented in Table 5 .

The overall morbidity rate was $52 \%$, with a significant difference between groups ( $100 \%$ vs. $41.9 \%$, respectively; $\mathrm{p}<0.001)$. The median operation times differed significantly between non-survivors and survivors ( 118 vs. 85 minutes; $\mathrm{p}=0.001$ ). Although the length of hospital stay was shorter in non-survivors than in the survivors $(p=0.027)$, the length of the ICU stay was significantly longer in non-survivors $(\mathrm{p}<0.001)$.

Overall, 184 complications occurred in 77 patients (51.3\%). In other words, 73 patients (48.7\%) had no complications. Surgical site infection and renal failure were the most common complications. Cardiac complications were observed only in patients who died. The types of complications are detailed in Table 6.

The univariate regression analysis identified frailty status, shock, and ASA grade as significant risk factors for mortality. In the multivariate regression analysis, ASA grade was the only independent risk factor for mortality (odds ratio $=19.642 ; 95 \%$ confidence interval, 3.886-99.274; $\mathrm{p}<0.001$ ) (Table 7).

\section{DISCUSSION}

The present retrospective study examined the effect of clinical factors and preoperative frailty on morbidity and mortality following emergency abdominal surgery in older patients. We found that $17.3 \%$ of patients died within 30 days of surgery. Older patients who were obese and frail with higher ASA grades were the most vulnerable to mortality following emergency abdominal surgery.

Given the increasing incidence of older patients requiring emergency laparotomy, there is an urgent need for a detailed understanding of the outcomes after this surgery because of the higher risks of mortality. ${ }^{2)}$ Consistent with previous studies, ${ }^{2,10,12,18,19)}$ the most significant predictors for mortality in the present study were age, ASA grade, frailty status, and presence of septic shock. It was not unexpected that these variables were associated with death as they represent the degree of preoperative physiological derange- 
Table 6. Distribution of complications in the study groups

\begin{tabular}{lccc}
\hline Complication & Overall $(\mathrm{n}=150)$ & Non-survivors $(\mathrm{n}=26)$ & Survivors $(\mathrm{n}=124)$ \\
\hline SSI & $45(24.5)$ & $11(12.5)$ & $34(35.4)$ \\
ARF & $30(16.3)$ & $11(12.5)$ & $19(19.8)$ \\
Sepsis & $23(12.5)$ & $20(22.7)$ & $3(3.1)$ \\
Respiratory & $20(10.9)$ & $4(4.5)$ & $16(16.7)$ \\
Cardiac & $16(8.7)$ & $16(18.2)$ & 0 \\
UTI & $14(7.6)$ & $1(1.1)$ & $13(13.5)$ \\
Bleeding & $11(6.0)$ & $8(9.1)$ & $3(3.1)$ \\
Evisceration & $10(5.4)$ & $8(9.1)$ & $2(2.1)$ \\
Anastomotic leakage & $8(4.3)$ & $4(4.5)$ & $4(4.2)$ \\
Stoma related & $7(3.8)$ & $5(5.7)$ & $2(2.1)$ \\
Total & 184 & 88 & 96 \\
\hline
\end{tabular}

Values are presented as number (\%).

SSI, surgical site infections; ARF, acute renal failure; UTI, urinary tract infection.

Table 7. Results of univariate and multivariate logistic regression analyses for mortality

\begin{tabular}{|c|c|c|c|c|c|c|c|c|}
\hline \multirow{3}{*}{ Variable } & \multicolumn{4}{|c|}{ Univariate } & \multicolumn{4}{|c|}{ Multivariate } \\
\hline & \multirow{2}{*}{ OR } & \multicolumn{2}{|c|}{$95 \% \mathrm{CI}$} & \multirow{2}{*}{ p-value } & \multirow{2}{*}{ OR } & \multicolumn{2}{|c|}{$95 \% \mathrm{CI}$} & \multirow{2}{*}{ p-value } \\
\hline & & Lower & Upper & & & Lower & Upper & \\
\hline Age ( $\geq 75$ vs. $65-74 y)$ & 0.005 & -0.098 & 0.105 & 0.943 & 0.772 & 0.115 & 5.193 & 0.790 \\
\hline BMI & 0.080 & -0.004 & 0.018 & 0.198 & 1.157 & 0.950 & 1.409 & 0.148 \\
\hline Hypertension (yes vs. no) & -0.038 & -0.132 & 0.071 & 0.553 & 0.711 & 0.134 & 3.773 & 0.689 \\
\hline $\mathrm{CAD}$ (yes vs. no) & 0.102 & -0.021 & 0.229 & 0.101 & 3.400 & 0.723 & 15.976 & 0.121 \\
\hline Frailty (yes vs. no) & 0.158 & 0.022 & 0.305 & 0.024 & 1.806 & 0.340 & 9.589 & 0.487 \\
\hline Septic shock (yes vs. no) & 0.268 & 0.165 & 0.475 & $<0.001$ & 4.040 & 0.499 & 32.723 & 0.191 \\
\hline ASA grade & 0.415 & 0.110 & 0.227 & $<0.001$ & 19.642 & 3.886 & 99.274 & $<0.001$ \\
\hline
\end{tabular}

BMI, body mass index; CAD, coronary artery disease; ASA, American Society of Anesthesiologists; OR, odds ratio; CI, confidence interval.

ment. Although there are controversial findings regarding the effect of advancing age on mortality, the mortality rates in the present study were higher in older patients aged $\geq 75$ years. Thus, like other investigators, $2,3,9,10,20)$ we recommend considering patient age in the decision-making process for emergency abdominal surgery in older patients.

The results of multivariate regression analysis in the present study identified ASA grade as the only independent risk factor for mortality. Although this finding was contrary to those of other studies in which the ASA grade was not associated with mortality after emergency colectomy, ${ }^{21,22)}$ this may be due to differences in population characteristics. Due to the possible reciprocal relationships between aging and morbidity, it is difficult to determine the specific effect of each factor on mortality. With increasing age, patients are more likely to have comorbidities and experience polypharmacy. ${ }^{19,23)}$ Hypertension and CAD were significant risk factors for the development of mortality in the present study. Therefore, physicians should be aware of the higher risk of adverse events leading to mortality in older patients with hypertension and CAD.
Frailty has been studied as a preoperative risk assessment variable to identify high-risk patients for mortality., ${ }^{3,24)}$ Although there is no universal definition of frailty, it may be defined as a phenotype that includes any combination of unintentional weight loss, self-reported exhaustion, grip strength weakness, slow walking speed, and low physical activity. ${ }^{4)}$ However, not all frail patients are old; thus, frailty can be considered a risk factor independent of age. $^{23,24)}$ Different scales or indices have been developed to assess frailty. ${ }^{4,6,17,23)}$ Many of these tools evaluate phenotypic measures such as physical strength, speed, activity, nutritional status, and fatigue. The main advantage of the mFI- 11 is its comorbidity-dominant scoring system. ${ }^{6}$ Because of the present study's retrospective design and unavailability of data to measure these phenotypic measures, we used the mFI-11 to assess frailty status. However, the clinical significance of each system has not been proven satisfactorily, and more research is needed to determine the optimum frailty assessment tool.

Previous studies have reported significant associations between frailty and several outcomes such as mortality, increased risk of 
complications, and length of hospital stay in older patients undergoing emergency laparotomy. ${ }^{3,17,18)}$ Frailty is also considered an independent risk factor for readmissions and post-discharge deaths. In the present study, frailty was also a significant risk factor for inpatient mortality during the postoperative 30 days. This result may be due to the use of the mFl-11 scale, which is a comorbidity-dominant scoring system. ${ }^{6}$ ) The results of the present study showed that higher ASA grades were significantly associated with mortality. Additionally, hypertension and CAD were predictive factors for mortality. Therefore, both the number and severity of comorbid diseases associated with frailty were crucial variables for the development of mortality in this patient group.

Multi-dimensional phenotypic manifestations of frailty, such as physical strength, walking speed, activity, nutritional status, and fatigue, could be better indicators of the physiological reserve. However, owing to the retrospective design of the present study, it was not possible to collect such data. The most common indication for surgical treatment in the present study was gastrointestinal perforation. Various etiological causes have been attributed to gastrointestinal perforation in different study populations, such as bowel obstruction secondary to cancer or adhesions. ${ }^{19)}$ Although the mortality rates vary based on the diagnoses, peritonitis is generally regarded as the most severe intra-abdominal pathology associated with high mortality rates. ${ }^{19)}$ However, we did not evaluate peritonitis as a separate diagnostic category in this study because there is some degree of subjectivity in grading peritonitis and the condition can result from various etiologies, such as perforation and ischemia.

The major limitation of the present study was that it assessed only 30 -day mortality and did not include 90-day mortality. The other limitations were the use of data from a single-center, retrospective design, lack of standardization for postoperative care protocols, and relatively small sample size.

In conclusion, older patients with obesity and frailty with higher ASA grades and septic shock had worse survival following emergency abdominal surgery. Early recognition of these high-risk groups necessitates dedicated and detailed follow-up after emergency abdominal surgery.

\section{ACKNOWLEDGMENTS}

\section{CONFLICT OF INTEREST}

The researchers claim no conflicts of interest.

\section{FUNDING}

None.

\section{AUTHOR CONTRIBUTION}

Conceptualization, NAH, AA; Data curation, YA, SM; Investigation, NAH, YU, MT, GE; Methodology, NAH, YU, MT, GE; Writing-original draft, NAH, TVA, OK; Writing-review \& editing, NAH, AA, YU, TVA, SM, MT, OK, GE, YA.

\section{REFERENCES}

1. Aburto JM, Villavicencio F, Basellini U, Kjaergaard S, Vaupel JW. Dynamics of life expectancy and life span equality. Proc Natl Acad Sci U S A 2020;117:5250-9.

2. Chua M, Chan D. Increased morbidity and mortality of emergency laparotomy in elderly patients. World J Surg 2020;44:71120.

3. Aitken RM, Partridge J, Oliver CM, Murray D, Hare S, Lockwood S, et al. Older patients undergoing emergency laparotomy: observations from the National Emergency Laparotomy Audit (NELA) years 1-4. Age Ageing 2020;49:656-63.

4. Sharrock AE, McLachlan J, Chambers R, Bailey IS, Kirkby-BottJ. Emergency abdominal surgery in the elderly: can we predict mortality? World J Surg 2017;41:402-9.

5. Ozban M, Birsen O, Senel M, Ozden A, Kabay B. The analysis of scoring systems predicting mortality in geriatric emergency abdominal surgery. Ulus Travma Acil Cerrahi Derg 2015;21:1826.

6. Tan HL, Chia S, Nadkarni NV, Ang SY, Seow DCC, Wong TH. Frailty and functional decline after emergency abdominal surgery in the elderly: a prospective cohort study. World J Emerg Surg 2019;14:62.

7. Ah R, BChir MB, Cao Y, Geijer H, Taha K, Pourhossein-Sarmeh S, et al. Prognostic value of P-POSSUM and osteopenia for predicting mortality after emergency laparotomy in geriatric patients. Bull Emerg Trauma 2019; 7:223-31.

8. Ukkonen M, Kivivuori A, Rantanen T, Paajanen H. Emergency abdominal operations in the elderly: a multivariate regression analysis of 430 consecutive patients with acute abdomen. World J Surg 2015;39:2854-61.

9. Liljendahl MS, Gogenur I, Thygesen LC. Emergency laparotomy in Denmark: a nationwide descriptive study. World J Surg 2020;44:2976-81.

10. Al-Temimi MH, Griffee M, Enniss TM, Preston R, Vargo D, Overton S, et al. When is death inevitable after emergency laparotomy? Analysis of the American College of Surgeons National Surgical Quality Improvement Program database. J Am Coll Surg 2012;215:503-11.

11. Mohil RS, Bhatnagar D, Bahadur L, Dev DK, Magan M. POSSUM and P-POSSUM for risk-adjusted audit of patients under- 
going emergency laparotomy. Br J Surg 2004;91:500-3.

12. Hunter Emergency Laparotomy Collaborator Group; Hunter Emergency Laparotomy Collaborator Group. High-risk emergency laparotomy in Australia: comparing NELA, P-POSSUM, and ACS-NSQIP calculators. J Surg Res 2020;246:300-4.

13. Toftlund SA, Gogenur I, Thygesen LC. Descriptive study of all Danish patients undergoing emergency exploratory laparotomies in the period 2003-2014. Scand J Public Health 2020; 48:243-9.

14. Havens JM, Columbus AB, Seshadri AJ, Brown C, Tominaga GT, Mowery NT, et al. Risk stratification tools in emergency general surgery. Trauma Surg Acute Care Open 2018;3: e000160.

15. Cao Y, Bass GA, Ahl R, Pourlotfi A, Geijer H, Montgomery S, et al. The statistical importance of P-POSSUM scores for predicting mortality after emergency laparotomy in geriatric patients. BMC Med Inform Decis Mak 2020;20:86.

16. Thahir A, Pinto-Lopes R, Madenlidou S, Daby L, Halahakoon C. Mortality risk scoring in emergency general surgery: are we using the best tool? J Perioper Pract 2021;31:153-8.

17. Juma S, Taabazuing MM, Montero-Odasso M. Clinical frailty scale in an acute medicine unit: a simple tool that predicts length of stay. Can Geriatr J 2016;19:34-9.

18. Goeteyn J, Evans LA, De Cleyn S, Fauconnier S, Damen C, Hewitt J, et al. Frailty as a predictor of mortality in the elderly emergency general surgery patient. Acta Chir Belg 2017; $117: 370-5$

19. Vilches-Moraga A, Rowley M, Fox J, Khan H, Paracha A, Price A, et al. Emergency laparotomy in the older patient: factors predictive of 12-month mortality-Salford-POPS-GS. An observational study. Aging Clin Exp Res 2020;32:2367-73.

20. Joseph B, Orouji Jokar T, Hassan A, Azim A, Mohler MJ, Kulvatunyou N, et al. Redefining the association between old age and poor outcomes after trauma: the impact of frailty syndrome. J Trauma Acute Care Surg 2017;82:575-81.

21. Oliver CM, Walker E, Giannaris S, Grocott MP, Moonesinghe SR. Risk assessment tools validated for patients undergoing emergency laparotomy: a systematic review. Br J Anaesth 2015; 115:849-60

22. Kwok AC, Lipsitz SR, Bader AM, Gawande AA. Are targeted preoperative risk prediction tools more powerful? A test of models for emergency colon surgery in the very elderly. J Am Coll Surg 2011;213:220-5.

23. Boyd-Carson H, Gana T, Lockwood S, Murray D, Tierney GM. A review of surgical and peri-operative factors to consider in emergency laparotomy care. Anaesthesia 2020;75 Suppl 1:e7582.

24. Kim DH. Measuring frailty in health care databases for clinical care and research. Ann Geriatr Med Res 2020;24:62-74. 